\title{
Comparing the washout profiles of desflurane in various anesthesia workstations
}

\author{
Yusuke Yamashita, Shinsuke Hamaguchi*, Makoto Komatsuzaki, Yoshiyuki Takahashi, and Toshihumi Takasusuki \\ Department of Anesthesia and Pain Medicine, Dokkyo Medical University School of Medicine, Japan
}

\begin{abstract}
Background: Malignant hyperthermia can be induced by halogenated anesthetics that are inadvertently retained in the anesthetic circuit. Therefore, we compared the desflurane washout times of three different anesthesia workstations.

Methods: The Aisys ${ }^{\mathrm{TM}}$, Fabius GS ${ }^{\mathrm{TM}}$, and Pro-next ${ }^{\mathrm{TM}}$ workstations were selected and each workstation was tested after connecting a 1-L test lung to the patient side and volume-controlled ventilation was performed with $500 \mathrm{~mL}$ tidal volume, a respiratory rate of $10 / \mathrm{min}$, and $\mathrm{O} 2 \mathrm{flow}$ of $1.5 \mathrm{~L} / \mathrm{min}$. When the end-tidal concentration of desflurane reached 6\%, the flow of desflurane was stopped and the time until desflurane had completely disappeared from the circuit under continuous gas flow was measured.

Results: The mean washout times were $175 \pm 5.5 \mathrm{~s}$ for the Aisys ${ }^{\mathrm{TM}}$ system, $216 \pm 13 \mathrm{~s}$ for the Fabius $\mathrm{GS}^{\mathrm{TM}}$ system, and $254 \pm 31 \mathrm{~s}$ for the Pro-next ${ }^{\mathrm{TM}}$ system. The calculated washout times per circuit capacity were $175 \pm 5.5 \mathrm{~s} / \mathrm{L}$ for the Aisys ${ }^{\mathrm{TM}}$ system, $216 \pm 13 \mathrm{~s} / \mathrm{L}$ for the Fabius GS ${ }^{\mathrm{TM}}$ system and $254 \pm 31 \mathrm{~s} / \mathrm{L}$ for the Pro-next ${ }^{\mathrm{TM}}$ system. The Aisys ${ }^{\mathrm{TM}}$ system provided significantly shorter times compared with the other systems.

Conclusion: The Aisys ${ }^{\mathrm{TM}}$ system provided the shortest washout time for desflurane. These findings may help prevent malignant hyperthermia among patients who are preparing to undergo anesthesia.
\end{abstract}

\section{Introduction}

Inhaled halogenated anesthetic agents are commonly used for general anesthesia. However, the time required for halogenated anesthetic agents to wash out of the anesthesia workstation, including the ventilator circuit, is an important safety consideration. Furthermore, the washout profiles of anesthesia workstations are useful for predicting patient recovery from general anesthesia. Moreover, halogenated anesthetics must be removed from the anesthesia workstation to prevent malignant hyperthermia. Several studies have investigated the kinetic profiles of various volatile anesthetics, such as sevoflurane or isoflurane [1]. However, Kim et al. [2] recently demonstrated that the Aisys $^{\mathrm{TM}}$ system (GE Healthcare, Madison, WI, USA), which uses an electronic vaporizer system, may retain saturated vapor in the breathing system. Few studies have evaluated the washout kinetics of desflurane, a new volatile anesthetic used in Japan, and no studies have evaluated the washout profiles of desflurane in different anesthetic machines. Although arousal from general anesthesia depends on the blood-gas partition coefficient values, patient factors, and anesthetic-specific factors, it is also possible that arousal depends on the respiratory and circulatory circuit volumes of the anesthesia machine. Therefore, the present study compared the washout profiles for desflurane in various anesthesia workstations that included different types of breathing systems and components.

\section{Materials and methods}

The present study evaluated non-patient-derived data; therefore, ethical approval was not required for this study. We evaluated the Aisys $^{\mathrm{TM}}$ (GE Healthcare), Fabius GSTM (Dräger, Inc., Lubick, German), and Pro-next ${ }^{\mathrm{TM}}$ (Acoma, Inc., Tokyo, Japan) anesthesia workstations, which are used in our operating theater and throughout Japan. The ventilators were tested using closed breathing circuits with a vaporizer of desflurane. Each circuit consisted of a 1-L test lung, a S5 Compact Gas Monitor TM (GE Healthcare), and a portable gas analyzer module (E-CAiOVX ${ }^{\mathrm{TM}}$; GE Healthcare). The workstations were tested after connecting the 1-L test lung to the patient side of the anesthesia circuit, and without a disposable humidifier. Volume-controlled ventilation was performed using $500 \mathrm{~mL}$ of tidal volume, a respiratory rate of $10 / \mathrm{min}$, and an $\mathrm{O}_{2}$ flow rate of $1.5 \mathrm{~L} / \mathrm{min}$. When the end-tidal concentration of desflurane reached $6 \%$ for $1 \mathrm{~min}$, the flow of desflurane was stopped and the time until desflurane had completely disappeared from the anesthetic circuit using continuous gas flow was measured. The total washout time for desflurane was measured using the $\mathrm{S} 5^{\mathrm{TM}}$ Compact Gas Monitor, which captures data every $5 \mathrm{~s}$. The mean and standard deviation values were calculated based on the results from 20 repetitions. Because each workstation has different breathing systems and components, we also calculated the total washout time divided by the total circuit volume. The total circuit volume was defined as the sum of the internal anesthesia breathing circuit, the volume of the soda lime canister, and the ventilator capacity including the respiratory bellows. The volumes for each workstation were obtained from the

Correspondence to: Shinsuke Hamaguchi, MD, PhD, Professor and Chairman, Department of Anesthesia and Pain Medicine, Dokkyo Medical University School of Medicine, 880 Kitakobayashi, Mibu, Tochigi 321-0293, Japan; Tel: +81282-86-1111 (ext. 2771); Fax: +81-282-86-0478; E-mail: s-hama@dokkyomed.ac.jp

Key words: washout time, halogenated inhaled anesthetics, desflurane, anesthesia workstation

Received: July 16, 2016; Accepted: August 10, 2016; Published: August 13, 2016 
leaflet provided by the manufacturer.

Results were expressed as the mean \pm standard error, and were compared using repeated measures analysis of variance with post hoc Bonferroni correction. All statistical analyses were performed using GraphPad Prism ${ }^{\mathrm{TM}}$ software (version 6.01; Graph Pad Software Inc., San Diego, CA, USA). All results were considered statistically significant at a $\mathrm{p}$-value $<0.05$.

\section{Results}

The specifications of each anesthesia workstation are shown in Table 1. The measured total washout times for desflurane were $902 \pm$ $28 \mathrm{~s}$ in the Aisys ${ }^{\mathrm{TM}}$ system, 1,060 $\pm 64 \mathrm{~s}$ in the Fabius GS ${ }^{\mathrm{TM}}$ system and $1,363 \pm 168 \mathrm{~s}$ in Pro-next ${ }^{\mathrm{TM}}$ system. The measured total washout time for desflurane in the Aisys ${ }^{\mathrm{TM}}$ was significantly shorter compared with the other two anesthesia workstations (both $\mathrm{p}<0.01$ ) (Figure 1). The calculated washout times per circuit capacity for desflurane were $175 \pm 5.5 \mathrm{~s} / \mathrm{L}$ in the Aisys ${ }^{\mathrm{TM}}$ system, $216 \pm 13 \mathrm{~s} / \mathrm{L}$ in the Fabius GS ${ }^{\mathrm{TM}}$ system, and $254 \pm 31$ s/L in the Pro-next ${ }^{\mathrm{TM}}$ system (Figure 2). The calculated washout time per circuit capacity was significantly shorter in the Aisys ${ }^{\mathrm{TM}}$ system compared with the Fabius GS ${ }^{\mathrm{TM}}$ and Pro-next ${ }^{\mathrm{TM}}$ systems (both $\mathrm{p}<0.01$ ). Moreover, the washout time in the Fabius GS ${ }^{\mathrm{TM}}$ system was significantly shorter than that in the Pro-next ${ }^{\mathrm{TM}}$ system ( $\mathrm{p}$ $<0.01)$.

\section{Discussion}

The present study revealed that the Aisys ${ }^{\mathrm{TM}}$ system provided a shorter desflurane washout time compared with the Fabius GS ${ }^{\mathrm{TM}}$ and Pro-next ${ }^{\mathrm{TM}}$ systems. Moreover, the washout time of the Fabius GS ${ }^{\mathrm{TM}}$

Table 1. Specifications for each anesthesia workstation

\begin{tabular}{|l|c|c|c|c|}
\hline Workstation & $\begin{array}{c}\text { Inside anesthesia } \\
\text { breathing circuit } \\
\text { (L) }\end{array}$ & $\begin{array}{c}\text { Soda lime } \\
\text { canister }(\mathbf{L})\end{array}$ & $\begin{array}{c}\text { Ventilator capacity } \\
\text { including the } \\
\text { respiratory } \\
\text { bellows (L) }\end{array}$ & $\begin{array}{c}\text { Total } \\
\text { capacity } \\
(\mathbf{L})^{\mathbf{a}}\end{array}$ \\
\hline Aisys $^{\mathrm{TM}}$ & 2.70 & 0.80 & 1.65 & 5.15 \\
\hline Fabius GSTM & 1.7 & 1.5 & 1.7 & 4.9 \\
\hline Pro-next & & 1.34 & 2.00 & 5.35 \\
\hline
\end{tabular}

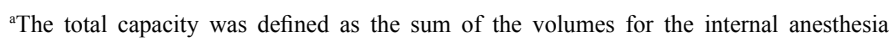
breathing circuit, the soda lime canister, and the ventilator including the respiratory bellows. All data were collected from the manufacturer's leaflet.

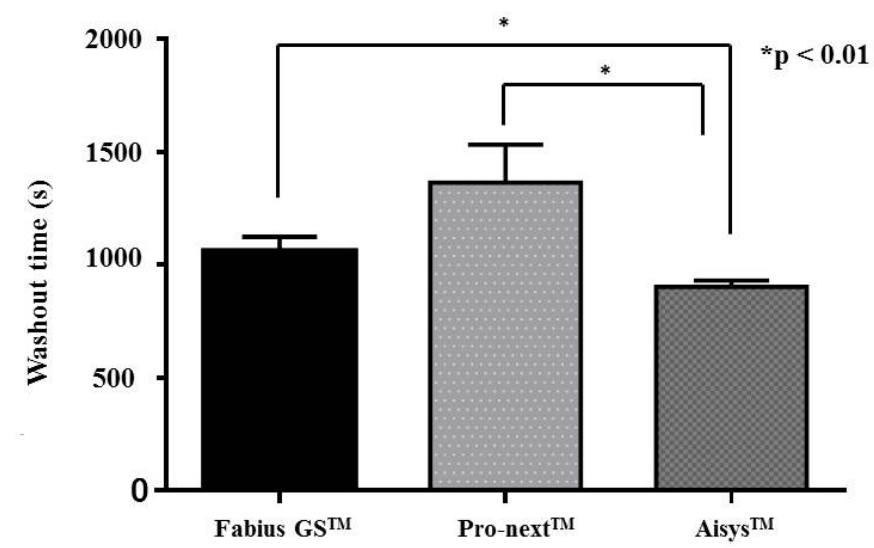

Figure 1. Comparison of the measured total washout times for desflurane

The measured total washout times for desflurane were $902 \pm 28 \mathrm{~s}$ in the Aisys ${ }^{\mathrm{TM}}$ system, $1,060 \pm 64 \mathrm{~s}$ in the Fabius GS ${ }^{\mathrm{TM}}$ system, and 1,363 $\pm 168 \mathrm{~s}$ in the Pro-next ${ }^{\mathrm{TM}}$ system. The total washout time for desflurane in the Aisys ${ }^{\mathrm{TM}}$ system was significantly lower than for the two other systems $(* p<0.01)$. Values are the mean \pm standard error.

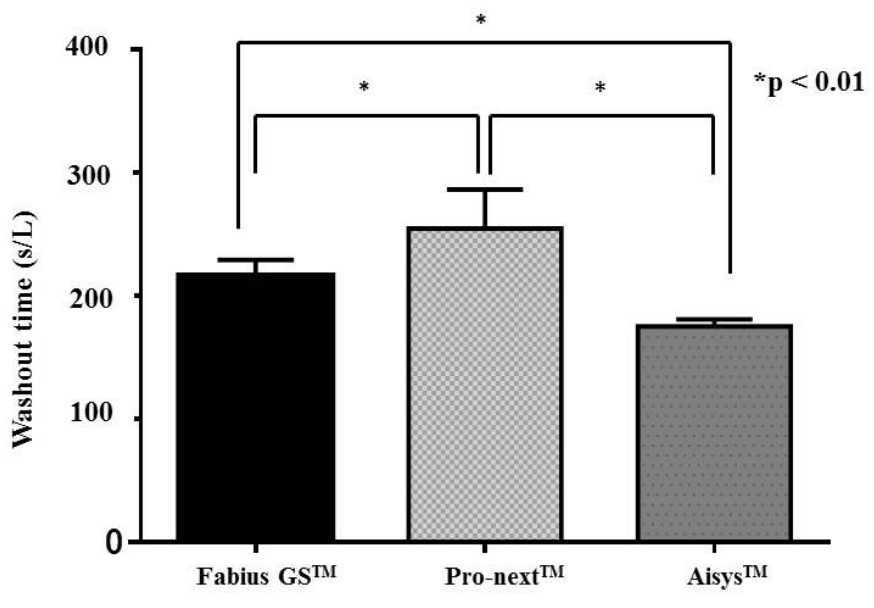

Figure 2. Comparison of the calculated washout times for desflurane

The calculated washout times for desflurane were $175 \pm 5.5 \mathrm{~s} / \mathrm{L}$ in the Aisys ${ }^{\mathrm{TM}}$ system, $216 \pm 13 \mathrm{~s} / \mathrm{L}$ in the Fabius GSTM system, and $254 \pm 31 \mathrm{~s} / \mathrm{L}$ in the Pro-next ${ }^{\mathrm{TM}}$ system. The calculated washout time per circuit capacity in the Aisys ${ }^{\mathrm{TM}}$ system was significantly shorter than in the Fabius GS ${ }^{\mathrm{TM}}(\mathrm{p}<0.01)$ and Pro-next ${ }^{\mathrm{TM}}$ systems (both $\left.\mathrm{p}<0.01\right)$. The calculated washout time per circuit capacity in the Fabius GS ${ }^{\mathrm{TM}}$ system was significantly shorter than that in the Pro-next ${ }^{\mathrm{TM}}$ system $(\mathrm{p}<0.01)$. Values are the mean \pm standard error.

system was significantly shorter than that of the Pro-next ${ }^{\mathrm{TM}}$ system. This information may be useful for preventing malignant hyperthermia among patients who are about to receive anesthesia, by ensuring complete desflurane washout in these workstations.

Varying concentrations are used for volatile anesthetic agents such as sevoflurane, isoflurane, or desflurane. Therefore, the patient's characteristics, duration and type of procedure, type of breathing system, and monitoring efficiency must be considered when selecting the optimal therapy for each patient [3]. Moreover, the minimum alveolar concentration of desflurane is higher than that for sevoflurane and isoflurane; therefore, given that each anesthetic workstation is produced by a different manufacturer, it is important to know the washout profiles for each workstation to ensure safe anesthetic management.

Several reports have described the kinetics of volatile anesthetics to prevent accidentally inducing malignant hyperthermia $[1,2,4-6]$. Cotton et al. [1] reported that the washout profiles of specific volatile agents may vary for different workstations. Furthermore, Kim et al. [2] reported that the Aisys ${ }^{\mathrm{TM}}$ system uses an electronic vaporizer system that may expose the breathing system to retained saturated vapor. Furthermore, Crawford et al. [4] suggested that the isoflurane washout rate was increased by 20 -fold for an autoclaved ventilator diaphragm and integrated breathing system. These reports indicated that workstation breathing systems may hinder the washout of gases. Therefore, we calculated the circuit volume for each workstation, and found that the Aisys ${ }^{\mathrm{TM}}$ system had the greatest flow capacity. However, the internal anesthesia breathing circuit is an important factor for anesthetic induction or washout [7]. Thus, we calculated the volumes for the various circuit components. The Fabius GSTM system had the largest soda lime canister volume, while the Pro-next ${ }^{\mathrm{TM}}$ system had the largest ventilator capacity and the largest total capacity. Therefore, we were unable to predict which system would provide the shortest washout time, and needed to perform actual testing for each workstation. 
This study had several limitations. First, we did not evaluate how components in each anesthesia workstation affected the washout time. Second, we did not use a fresh-gas flush to remove desflurane. Usually, many anesthesiologists use the fresh-gas flush for wash out the volatile agents but rather select continuous gas flow to wash out the halogenated volatile agent to prepare the workstation for patients who cannot be exposed to desflurane.

\section{Conclusions}

In conclusion, we evaluated the washout times for desflurane in various anesthesia workstations. Our results indicate that the Aisys ${ }^{\mathrm{TM}}$ system washes out desflurane quicker than the Fabius GSTM and Pronext ${ }^{\mathrm{TM}}$ systems, based on continuous gas flow. However, other studies and washout conditions are required to validate our findings.

\section{References}

1. Cottron N, Larcher C, Sommet A, Fesseau R, Alacoque X, et al. (2014) The sevoflurane washout profile of seven recent anesthesia workstations for malignant hyperthermiasusceptible adults and infants: a bench test study. Anesth Analg 119: 67-75. [Crossref]
2. Kim TW, Wingate JR, Fernandez AM, Whitaker E, Tham RQ (2013) Washout times of desflurane, sevoflurane and isoflurane from the GE Healthcare Aisys ${ }^{\circledR}$ and Avance ${ }^{\circledR}$, Carestation $^{\circledR}$, and Aestiva ${ }^{\circledR}$ anesthesia system. Paediatr Anaesth 23: 1124-1130. [Crossref]

3. Sakai EM, Connolly LA, Klauck JA (2005) Inhalation anesthesiology and volatile liquid anesthetics: focus on isoflurane, desflurane, and sevoflurane. Pharmacotherapy 25:1773-1788. [Crossref]

4. Crawford MW, Prinzhausen H, Petroz GC (2007) Accelerating the washout of inhalational anesthetics from the Dräger Primus anesthetic workstation: effect of exchangeable internal components. Anesthesiology 106: 289-294. [Crossref]

5. Brünner HW, Pohl S, Grond S (2011) Washout of sevoflurane from the GE Avance and Amingo Carestation anesthetic machines. Acta Anaesthesiol Scand 55: 1118-1123. [Crossref]

6. Struys MM, Kalmar AF, De Baerdemaeker LE, Mortier EP, Rolly G, et al. (2005) Time course of inhaled anaesthetic drug delivery using a new multifunctional closed-circuit anaesthesia ventilator. In vitro comparison with a classical anaesthesia machine. $\mathrm{Br} J$ Anaesth 94: 306-317. [Crossref]

7. Kern D, Larcher C, Basset B, Alacoque X, Fesseau R, et al. (2012) Inside anesthesia breathing circuits: time to reach a set sevoflurane concentration in toddlers and newborns: simulation using a test lung. Anesth Analg 115:310-314. [Crossref]

Copyright: $@ 2016$ Yamashita Y. This is an open-access article distributed under the terms of the Creative Commons Attribution License, which permits unrestricted use, distribution, and reproduction in any medium, provided the original author and source are credited. 